\title{
MODELLING TEMPORAL SCHEDULE OF URBAN TRAINS USING AGENT-BASED SIMULATION AND NSGA2-BASED MULTIOBJECTIVE OPTIMIZATION APPROACHES
}

\author{
Mohammadreza Sahelgozin ${ }^{\text {a }}$,Abbas Alimohammadi ${ }^{\text {b }}$ \\ GIS Dept., Geoinformation Technology Center of Excellence, Faculty of Geodesy\&Geomatics Engineering \\ K.N.Toosi University of Technology, Tehran, Iran \\ a sahelgozin@mail.kntu.ac.ir_b alimoh_abb@kntu.ac.ir
}

KEY WORDS: Subway Systems, Temporal Schedule, Modelling, Agent-based Simulation, Multi-objective Optimization

\begin{abstract}
:
Increasing distances between locations of residence and services leads to a large number of daily commutes in urban areas. Developing subway systems has been taken into consideration of transportation managers as a response to this huge amount of travel demands. In developments of subway infrastructures, representing a temporal schedule for trains is an important task; because an appropriately designed timetable decreases Total passenger travel times, Total Operation Costs and Energy Consumption of trains. Since these variables are not positively correlated, subway scheduling is considered as a multi-criteria optimization problem. Therefore, proposing a proper solution for subway scheduling has been always a controversial issue. On the other hand, research on a phenomenon requires a summarized representation of the real world that is known as Model. In this study, it is attempted to model temporal schedule of urban trains that can be applied in Multi-Criteria Subway Schedule Optimization (MCSSO) problems. At first, a conceptual framework is represented for MCSSO. Then, an agent-based simulation environment is implemented to perform Sensitivity Analysis (SA) that is used to extract the interrelations between the framework components. These interrelations is then taken into account in order to construct the proposed model. In order to evaluate performance of the model in MCSSO problems, Tehran subway line no. 1 is considered as the case study. Results of the study show that the model was able to generate an acceptable distribution of Pareto-optimal solutions which are applicable in the real situations while solving a MCSSO is the goal. Also, the accuracy of the model in representing the operation of subway systems was significant.
\end{abstract}

\section{INTORDUCTION}

Urban growth results in a significant increase in the distances between locations of people's residence and locations of services such as education, occupation, shopping, health and recreation. This leads to a large number of daily commutes in urban areas; usually from home to work or school in early morning hours and in the opposite direction in the evenings. A suitably developed transportation infrastructure is a requirement to satisfy the huge amount of inter-cities travel demands. Nowadays, urban rail transportation systems (Subway Systems) are under consideration of urban transportation managers. Consumption of non-fossil fuels, reduction of the general operation costs as well as reliability and predictability of the travel times are significant advantages of the subway systems. Therefore, developments of the urban subway infrastructures are vital to improve satisfaction of both the transportation managers and passengers.

In addition to the optimization of the rail route and locations of stations, design of a temporal schedule for train operations is an important task for effective use of the system. An appropriately designed timetable would certainly improves the quality of service in subway systems (Yang et al., 2014). Three common criteria including Total passenger travel times, Total Operation Costs and Energy Consumption of trains are in the main concentration of the policy makers as the service quality indicators. There are two-sided interrelations that exist between each of these criteria with the temporal schedule of subway systems. For example, total passenger travel times is considered as an effective factor in the timetable design process; on the other hand, total passenger travel times is affected by the timetable too.
Similarly, total operation costs and energy consumption of trains have also the same interrelations with the temporal schedule of trains.

Furthermore, these criteria are not positively correlated with each other. Therefore, train scheduling is considered as a multiobjective optimization problem (Chang et al., 2000). Therefore, proposing a proper solution for subway scheduling that simultaneously optimizes all of the mentioned criteria has been always a controversial issue. However, finding a suitable solution for a phenomenon requires a perception of the phenomenon that can be gotten by a generalized representation of the real world. This representation is achieved with the help of a Modell.

In this study, it is attempted to model temporal schedule of urban trains operation in a way that it could be applied in Multi-Criteria Subway Schedule Optimization (MCSSO) problems. In order to accomplish this, a conceptual framework is designed in Section 3. The parameters that are related to subway scheduling are represented in the framework. These parameters are extracted by reviewing the literature. Then, an agent-based simulation environment is implemented in Section 4 that is a tool for performing further Sensitivity Analysis (SA). In this study, SA is used for understanding interrelations between the proposed framework components as well as their influences on the service quality indicators. In section 5, interrelations between the framework components in addition to a number of basic laws of physics are summarized in a set of mathematical functions which together represent a model for temporal schedule of subway systems. In order to evaluate performance of the developed model and how it can help to solve MCSSO problems, Tehran

\footnotetext{
* Corresponding author
} 
subway line no. 1 is considered as the case study. By feeding the proposed model with the real data, the evaluation results are obtained that are provided in Section 6. Finally, conclusion of the study is provided in Section 7.

\section{RELATED WORKS}

A large number of researches has been performed in scheduling of transportation systems in the recent years. Scheduling passenger trains and urban trains are a major part of these studies. The large number of studies in this scope is because of the complexities of trains scheduling problems. In this regard, Yang et al. (2014) believed that physical complexities of subway systems is the reason that subway scheduling is one of the most controversial issues.

Apart from initial solutions for trains scheduling which were usually manual, other studies which concentrated on the problems that are similar to multi-criteria train scheduling optimization are summarized in this section. In a number of studies, schedule optimization of trains was performed so that it reduces energy consumption or energy loss. They include proposing an algorithm which distributes travel times of the trains for the most efficient energy consumption (Su et al., 2013), developing a cooperative scheduling model to increase simultaneous accelerates and brakes of the consecutive trains (Nasri et al., 2010; Yang et al., 2013) and applying the genetic algorithm to decrease the simultaneous acceleration of trains in order to avoid maximum traction power of the system (Chen et al., 2005).

Furthermore, some other studies focused on the multi-objective optimization of timetables for transportation systems in order to satisfy some criteria. Decreasing travel time and operation costs in high-speed rail systems (Chang et al., 2000), travel time and energy consumption in passenger trains (Ghoseiri et al., 2004; Chevrier et al., 2013; Hu et al., 2013), operation costs and energy consumption in a freight transportation system (Lau et al., 2013) and decreasing travel time, operation costs and energy consumption in a sustainable road network design problem (Kim et al., 2012) are some examples in this scope.

In addition, there were some attempts in developing models and approches for trains scheduling. Shaoquan et al. (2009) represented an optimization model for initial schedule of passenger trains that is based on an improved genetic algorithm. Also, Sels et al. (2013) introduced an objective function for the total passenger travel times in subway systems in order to overcome the lack of mathematical modelling in a sustainable scheduling. Furthermore, Chang and Kwan (2005) evaluated the performance of the evolutionary algorithms including Genetic Algorithm (GA), Particle Swarm Optimization (PSO) and Differential Evolution (DE) in trains schedule optimization problems.

\section{SUBWAY SCHEDULE OPTIMIZATION}

In this section, a conceptual framework is proposed for MCSSO that aggregates all the parameters which are related to the operation of subway systems. Structure of the proposed framework is inspired from the concept of Sustainable Transportation. With regard to the definition of Sustainable Development, three components including Social Equity, Economic Efficiency and Environmental Quality of the system should be considered in sustainable transportation planning (Kim et al., 2012). For each of these components, a corresponding category is considered in the MCSSO framework: Passengers, Operation Company and Environment. Then, the related parameters to the subway scheduling that are extracted by literature review are grouped in these three categories (Figure 1).

Apart from categorization of the parameters, it is necessary for their interrelations to be represented in the framework. The interrelations are extracted by the sensitivity analysis and are provided in Table 1. In this table, the fact that if two parameters are correlated (positively or negatively) or not is shown.

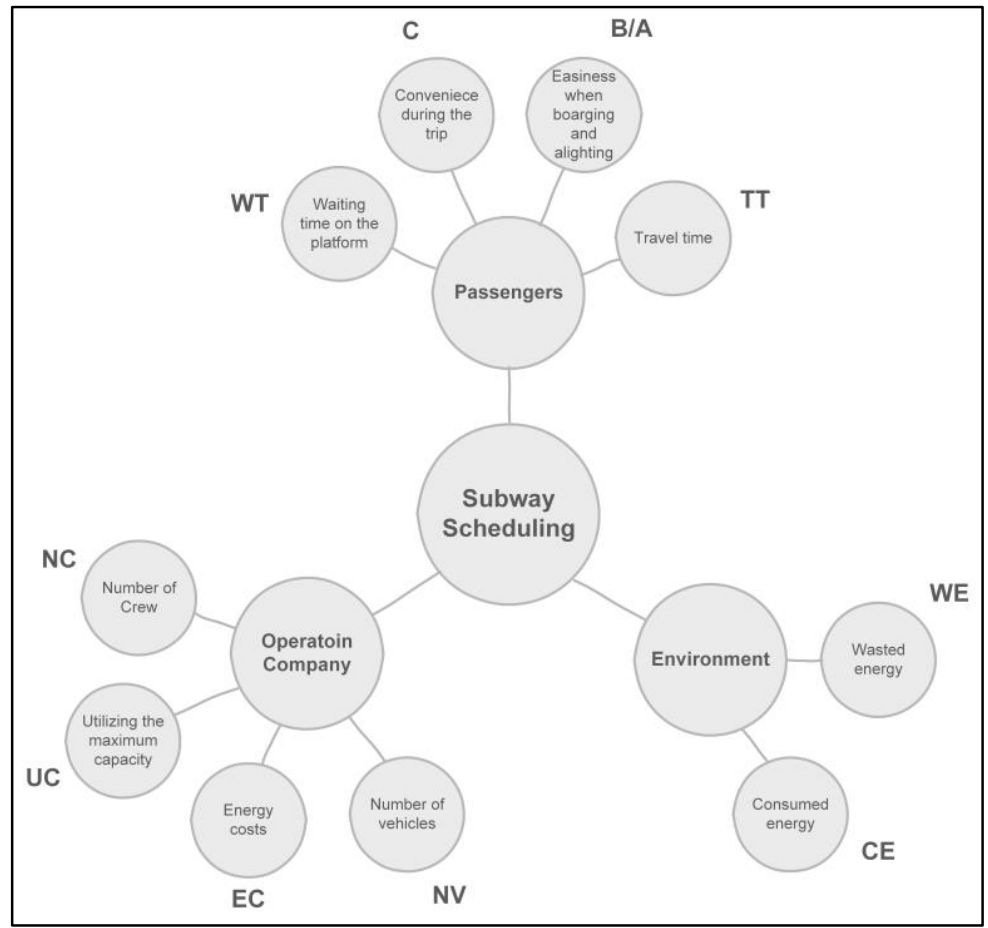

Figure 1. A conceptual framework for representing the parameters of MCSSO 
Table 1. Interrelations between the proposed framework parameters

\begin{tabular}{|c|c|c|c|c|c|c|c|c|c|c|c|}
\hline & \multicolumn{4}{|c|}{ Passengers } & \multicolumn{4}{|c|}{ Operation company } & \multicolumn{2}{|c|}{ Environment } \\
\hline & & WT & $\mathbf{C}$ & B/A & TT & NV & NC & $\mathbf{U C}$ & EC & $\mathbf{C E}$ & WE \\
\hline \multirow{4}{*}{ Passengers } & WT & $\begin{array}{ll}--- \\
\end{array}$ & ---- & $\begin{array}{ll}--- \\
\end{array}$ & + & - & - & + & - & - & - \\
\hline & $\mathbf{C}$ & $\begin{array}{ll}--- \\
\end{array}$ & $\begin{array}{ll}--- \\
\end{array}$ & + & - & + & + & - & + & + & + \\
\hline & B/A & ---- & + & ---- & ---- & + & + & - & + & + & + \\
\hline & TT & + & - & $\begin{array}{ll}--- \\
\end{array}$ & $\begin{array}{ll}--- \\
\end{array}$ & - & - & + & - & - & - \\
\hline \multirow{4}{*}{$\begin{array}{l}\text { Operation } \\
\text { company }\end{array}$} & NV & - & + & + & - & ---- & + & ---- & + & + & + \\
\hline & $\mathrm{NC}$ & - & + & + & - & + & ---- & ---- & ---- & ---- & ---- \\
\hline & $\mathbf{U C}$ & + & - & - & + & ---- & ---- & ---- & ---- & ---- & ---- \\
\hline & EC & - & + & + & - & + & ---- & ---- & ---- & + & + \\
\hline \multirow{2}{*}{ Environment } & CE & - & + & + & - & + & ---- & ---- & + & ---- & + \\
\hline & WE & - & + & + & - & + & ---- & ---- & + & + & ---- \\
\hline
\end{tabular}

\section{AGENT-BASED SIMULATION}

In this study, the simulation environment is implemented in MATLAB 2013 in which each agent is defined as a matrix. Rows of the matrix for an agent, represent instances of that agent. The instances are identified by a unique ID number. On the other hand, each column shows a real-time state or behaviour of the instances. Table 2 represents the designed agents including their states and behaviours. Also, the permitted values of their states and behaviours are provided in the table.

In the general procedure of the simulation, there is a variable which is named as "timeStep" whose job is to count the seconds. A main loop runs iteratively that corresponds to the seconds and updates states of the agents in each second. For example, in each iteration, the values for location, velocity and acceleration of the trains are updated according to the trains' actions and some physics formulas.

In addition, some service quality indicators which are defined in the simulation environment (Eq.1) are updated in the main loop as well. This helps us to get an estimation of the service quality at the end of the simulation procedure with which the performance of the system could be assessed.

$$
\begin{aligned}
& I_{\text {Passenger }}=\sum_{p=1}^{P} T T_{p} \\
& I_{\text {Cost }}=\sum_{n=1}^{N} \sum_{i=1}^{I} T o T_{i}^{n, n+1} *\left(I * C_{T}-P_{i}^{n, n+1}\right) \\
& I_{\text {Energy }}=\sum_{i=1}^{I} E_{i}
\end{aligned}
$$

Where $N, I$ and $P$ are the numbers of stations, trains and passengers, respectively. In addition, $C_{T}$ is the capacity of each train. $T T_{p}$ is the travel time for the passenger $p$ while $T o T_{i}^{n, n+1}$ and $P_{i}^{n, n+1}$ are the travel time and the number of boarded passengers of the train $i$ between the stations $n$ and $n+1$. Also,

\begin{tabular}{|c|c|c|}
\hline \multicolumn{3}{|c|}{ Stations } \\
\hline Specification of the Agent & Type & Permitted Values \\
\hline $\begin{array}{l}\text { Incoming passengers } \\
\text { rate (east platform) }\end{array}$ & State & $N^{*}$ \\
\hline $\begin{array}{l}\text { Outgoing passengers } \\
\text { rate (east platform) }\end{array}$ & State & $N$ \\
\hline $\begin{array}{l}\text { Incoming passengers } \\
\text { rate (west platform) }\end{array}$ & State & $N$ \\
\hline $\begin{array}{l}\text { Outgoing passengers } \\
\text { rate (west platform) }\end{array}$ & State & $N$ \\
\hline $\begin{array}{c}\text { Number of waiting } \\
\text { passengers (east } \\
\text { platform) }\end{array}$ & State & $N$ \\
\hline $\begin{array}{l}\text { Number of waiting } \\
\text { passengers (west } \\
\text { platform) }\end{array}$ & State & $N$ \\
\hline \multicolumn{3}{|c|}{ Trains } \\
\hline Specification of the Agent & Type & Permitted Values \\
\hline Location & State & $\begin{aligned} & \left(0, \text { max }_{\text {Location }}\right) \\
\in & R^{* *}\end{aligned}$ \\
\hline Speed & State & $\left(0, \max _{\text {Speed }}\right) \in R$ \\
\hline Acceleration & State & $\begin{array}{l}\left(0, \text { max }_{\text {Acceleration }}\right) \\
\in R\end{array}$ \\
\hline $\begin{array}{c}\text { Number of the boarded } \\
\text { passengers }\end{array}$ & State & $N$ \\
\hline Consumed energy & State & $\{R \geq 0\}$ \\
\hline $\begin{array}{l}\text { ID of the last departed } \\
\text { station }\end{array}$ & State & $\{$ Stations IDs\} \\
\hline Current action & Behaviour & $\begin{array}{c}\{1=\text { Acceleration } \\
2=\text { Coasting } \\
3=\text { Braking } \\
4=\text { Dwelling }\}\end{array}$ \\
\hline $\begin{array}{l}\text { The time remained for } \\
\text { the current action }\end{array}$ & State & $N($ Seconds $)$ \\
\hline Direction of the movement & State & $\{-1,1\}$ \\
\hline \multicolumn{3}{|c|}{ Passengers } \\
\hline Specification of the Agent & Type & Permitted Values \\
\hline Current Action & Behaviour & $\begin{array}{c}\{0=\text { Not traveling } \\
1=\text { Traveling }\}\end{array}$ \\
\hline Start time of the trip & State & $N($ Seconds $)$ \\
\hline ID of the origin station & State & $\{$ Stations IDs $\}$ \\
\hline ID of the last passed station & State & $\{$ Stations IDs \\
\hline ID of the destination station & State & $\{$ Stations IDs $\}$ \\
\hline ID of the carrier train & State & $\{$ Trains $I D s\}$ \\
\hline Travel time until now & State & $N($ Seconds $)$ \\
\hline
\end{tabular}
$E_{i}$ is the consumed energy of the train $i$.
Table 2. The designed agents in the simulation environment 


\section{THE PROPOSED MODEL}

\subsection{Decision Variables}

In addition to extracting interrelations between the parameters in the proposed framework (Section 3), the implemented agentbased simulation environment was also used to choose appropriate Decision Variables for MCSSO. The simulation was performed in a number of scenarios in each of which the value of a specific variable is changed significantly. Those variables that caused more clear changes in the service quality indicators were chosen as decision variables (Table 3 ).

Table 3. Decision Variables

\begin{tabular}{|c|c|}
\hline $\begin{array}{c}\text { Parame of acceleration for the train } i \text { between the } \\
\text { stations } n \text { and } n+1\end{array}$ & Notation \\
\hline $\begin{array}{c}\text { Time of braking for the train } i \text { between the } \\
\text { stations } n \text { and } n+1\end{array}$ & $T o A_{i}^{n, n+1}$ \\
\hline Time of dwell for the train $i$ in the station $n$ & $T o D_{i}^{n, n+1}$ \\
\hline $\begin{array}{c}\text { Acceleration rate for the train } i \text { between the } \\
\text { stations } n \text { and } n+1\end{array}$ & $a_{i}^{n, n+1}$ \\
\hline $\begin{array}{c}\text { Braking acceleration rate for the train } i \text { between } \\
\text { the stations } n \text { and } n+1\end{array}$ & $b_{i}^{n, n+1}$ \\
\hline
\end{tabular}

\subsection{Mathematical Objective Functions}

The developed objective functions for Total passenger travel times $\left(F_{1}\right)$, Total Operation Costs $\left(F_{2}\right)$ and Energy Consumption $\left(F_{3}\right)$ are represented in Eq. 2. Parameters of the model is explained in Tables 3 and 4. It should be noted that the total operation costs is considered based on the amount of the trains' capacities which is remained empty between the stations. Therefore, $F_{2}$ is the aggregated number of total boarded passengers that is going to be maximized. On the other hand, $F_{1}$ and $F_{3}$ should be minimized.

$$
\begin{array}{rl}
F_{1}=\sum_{i=1}^{I} \sum_{n=1}^{N-1}\left\{\frac{1}{2} * B_{i}^{n} *\right. & T o H_{i, i+1}^{n}+\frac{d_{n, n+1}}{a_{i}^{n, n+1} * T o A_{i}^{n, n+1}}+\frac{1}{2} \\
& \left.*\left(T o A_{i}^{n, n+1}+T o B_{i}^{n, n+1}\right)+T o D_{i}^{n}\right\} \\
F_{2}=\sum_{i=1}^{I} \sum_{n=1}^{N-1} B_{i}^{n} * T o D_{i}^{n} \\
F_{3}=\sum_{i=1}^{I} \sum_{n=1}^{N-1}\left(M+m * \frac{C_{T}}{2}\right) \\
*\left[\frac{1}{2}\left(a_{i}^{n, n+1}+f_{k} * g\right) * a_{i}^{n, n+1}\right. \\
\left.*\left(T o A_{i}^{n, n+1}\right)^{2}\right)+f_{k} * g * a_{i}^{n, n+1} \\
* & T o A_{i}^{n, n+1} * T o C_{i}^{n, n+1} \\
& +\left(b_{i}^{n, n+1}-f_{k} * g\right) * T o B_{i}^{n, n+1} *\left(\frac{1}{2}\right. \\
& \left.\left.* T o B_{i}^{n, n+1}+a_{i}^{n, n+1} * T o A_{i}^{n, n+1}\right)\right]
\end{array}
$$

\subsection{Model Constraints}

Some constraints are required to be taken into account in order to avoid model failures. Eq. 3 shows the considered constraints in the model. In addition to Eq. 3, some other constraints are defined as the lower and upper boundaries of the decision variables.

$$
\begin{array}{ll}
\text { - } & a_{i}^{n, n+1} * T o A_{i}^{n, n+1} \leq V_{\max } \\
\text { - } & a_{i}^{n, n+1} * T o A_{i}^{n, n+1}=-b_{i}^{n, n+1} * T o B_{i}^{n, n+1} \\
\text { - } & \frac{1}{2} a_{i}^{n, n+1}\left(T o A_{i}^{n, n+1}\right)^{2}+\frac{1}{2} b_{i}^{n, n+1}\left(T o B_{i}^{n, n+1}\right)^{2}+ \\
& \left(a_{i}^{n, n+1} * T o A_{i}^{n, n+1}\right) * T o B_{i}^{n, n+1} \leq d_{n, n+1}
\end{array}
$$

Where $V_{\max }$ is the maximum speed of the trains.

Table 4. Definition of the Model Parameters

\begin{tabular}{|c|c|}
\hline Parameter & Notation \\
\hline Number of stations & $N$ \\
\hline Distance between the stations $n$ and $n+1$ & $d_{n, n+1}$ \\
\hline Number of the active trains & $I$ \\
\hline Average weight of each train & $M$ \\
\hline Average weight of a passenger & $m$ \\
\hline $\begin{array}{c}\text { Time of the move at a constant speed for the } \\
\text { train } i \text { between the station } n \text { and } n+1\end{array}$ & $T o C_{i}^{n, n+1}$ \\
\hline $\begin{array}{c}\text { Headway time between the two consecutive } \\
\text { trains } i \text { and } i+1 \text { in the station } n\end{array}$ & $T_{o} H_{i, i+1}^{n}$ \\
\hline $\begin{array}{c}\text { Boarding passengers rate for the train } i \text { in the } \\
\text { station } n\end{array}$ & $B_{i}^{n}$ \\
\hline Capacity of each train & $C_{T}$ \\
\hline $\begin{array}{c}\text { Coefficient of kinetic friction between the train } \\
\text { wheels and the rail }\end{array}$ & $f_{k}$ \\
\hline Gravitational acceleration & $g$ \\
\hline
\end{tabular}

\section{EVALUATION OF THE PROPOSED MODEL}

\subsection{Case Study}

The proposed model was tested in Tehran subway line no.1 as the case study (Figure 2). Geospatial data as well as the descriptive data were gathered in a geo-dataset with the help of official Tehran GIS data, data of Tehran Urban and Suburban Railway Operation Company, Google Earth and a field survey. In addition, data for the current scheduling of Tehran subway line no.1 were obtained by the current timetable and also by measuring average time of acceleration, dwell or braking of the trains in the field survey.

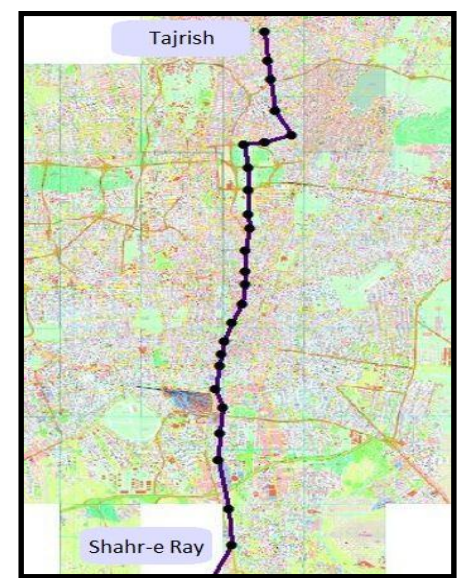

Figure 2. Study Area 


\subsection{Assumptions}

In the optimization process, some assumptions was considered for simplifications. It is assumed that operation of all the trains are similar between each two specific stations.

\subsection{Optimization Procedure: Results and Discussions}

An effective approach to deal with the multi-objective problems is optimization with the multi-objective evolutionary algorithms. Also, one of the most beneficial solutions for trains schedule optimizations are the Pareto-based methods (Ghoseiri et al., 2004; Chang and Kwan, 2005). Therefore, in this study, NSGAII (Deb et al., 2002) is used to solve the subway scheduling problem.

Using NSGA-II, a large number of solutions were obtained for the decision variables of the proposed model. To decrease the number of output data, Fuzzy Subtractive Clustering method was used to cluster the solutions. This method has two advantages: first, centre of the clusters are coincident on the Pareto front; second, the clusters are not crisply discrete. The radius of influence was set to 0.42 in the clustering process. The clustered solutions which were considered as the candidate schedules are provided in Table 5. Objective functions for the current schedule of Tehran subway line no. 1 is also represented in the table for further comparisons.

Table 5. Objective Functions for the candidate schedules in comparison to the current schedule

\begin{tabular}{|c|c|c|c|}
\hline \multirow{2}{*}{ Solution } & \multicolumn{3}{|c|}{ Objective Functions } \\
\cline { 2 - 4 } & Time $\left(10^{3}\right)$ & Cost $\left(10^{4}\right)$ & Energy $\left(10^{10}\right)$ \\
\hline 1 & 8.521 & -2.563 & 1.087 \\
\hline 2 & 7.355 & -3.116 & 1.094 \\
\hline 3 & 7.752 & -2.820 & 1.091 \\
\hline 4 & 1.671 & -4.710 & 1.944 \\
\hline 5 & 2.018 & -6.056 & 1.838 \\
\hline 6 & 6.045 & -3.580 & 1.111 \\
\hline 7 & 6.594 & -3.806 & 1.107 \\
\hline 8 & 1.889 & -5.799 & 1.832 \\
\hline 9 & 5.385 & -3.716 & 1.126 \\
\hline 10 & 1.902 & -5.496 & 1.757 \\
\hline 11 & 4.872 & -4.287 & 1.148 \\
\hline Current & 3.115 & -4.656 & 1.286 \\
Schedule & & \multicolumn{2}{|c}{} \\
\hline
\end{tabular}

To obtain a suitable vision, data in the above table are drawn in Figure 3. It is important to say that the objective functions of the candidate solutions has been normalized before they are drawn in order to avoid heterogeneity in the scales of the data. Each coloured line in the figure represents one of the cluster centers in the Table 5 .

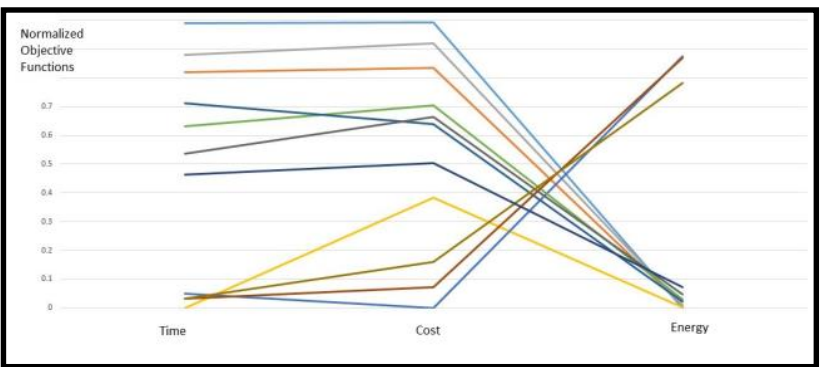

Figure 3.Normallized Objective Functions for the Candidate schedules
To choose the preferred schedule amongst the candidate schedules, it is required to consider preferences of the policy makers. Weighting the objective functions is the common way in this scope. In this study, Analytic Hierarchy Process (AHP) (Saaty, 1990) was applied as the weighting method. With regard to the obtained weights by the AHP method, the preferred schedule with the least weighted summation of the objective functions were chosen. The preferred schedule is compared with the current schedule in Table 6 . The trains' speed-time graph for both schedules are represented in Figure 4.

Table 6. The Preferred Schedule

\begin{tabular}{|c|c|c|c|}
\hline \multirow{2}{*}{ Solution } & \multicolumn{3}{|c|}{ Objective Functions } \\
\cline { 2 - 4 } & $\begin{array}{c}\text { Time } \\
\left(10^{3}\right)\end{array}$ & $\begin{array}{c}\text { Cost } \\
\left(10^{4}\right)\end{array}$ & $\begin{array}{c}\text { Energy } \\
\left(10^{10}\right)\end{array}$ \\
\hline Preferred Schedule & 2.922 & -5.126 & 1.365 \\
\hline Current Schedule & 3.115 & -4.656 & 1.286 \\
\hline Percentage Change & $-6.20 \%$ & $-10.09 \%$ & $+6.14 \%$ \\
\hline
\end{tabular}

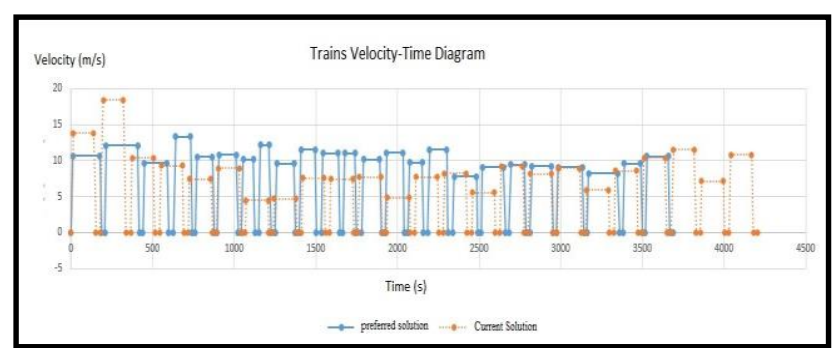

Figure 4. The Speed-Time Graph for the Preferred Schedule in comparison to the Current Schedule

As can be seen, the preferred schedule decreased the total passenger travel times and total operation costs by 6.2 and 10.1 , respectively. This is the reason that the preferred schedule in Figure 4 (the blue solid line) took less time for the trains to travel from the origin station to the last station in comparison to the current schedule (the red dashed line).

\section{CONCLUSION}

The objective of this study was to propose a mathematical model for temporal schedule of urban trains. The motivation of developing such a model was to help the process of Multi-Criteria Subway Schedule Optimization (MCSSO). First, a conceptual framework was proposed that represents the parameters which are related to the subway schedule. Next, an agent-based simulation environment was developed to perform sensitivity analysis in order to extract the interrelations between the framework components. Then, by the help of the developed framework, a mathematical model for MCSSO was created. The decision variables and the objective functions in the model were defined in a way that they could be applied in a MCSSO process.

Tehran subway line no.1 was considered as the case study to evaluate the performance of the proposed model. Operation of the system was modelled mathematically and multi-criteria optimization of its schedule was performed. Results of the evaluation show that using the suggested model outputted an acceptable distribution of Pareto solutions. Comparing the objective functions of the preferred schedule with those of the current schedule shows reductions in the total passenger travel times and total operation costs by 6.2 and 10.1 percent, respectively. By reviewing the results, the efficiency of the proposed model can be concluded. In addition, it is provable that the developed agent-based simulation was successful in the sensitivity analysis procedure. 
However, some limitations have remained with the proposed model. The model may not be compatible with the real-time data and it needs more flexibility and adaptations for instantaneous decision makings. Therefore, improving the model so that it can be compatible with the real-time data would be considerable as the future work.

\section{REFERENCES}

Chang, C. S. and Kwan, C. M., 2005. Evaluation of evolutionary algorithms for multi-objective train schedule optimization. $A I$ 2004: Advances in Artificial Intelligence, Springer: 803-815.

Chang, Y.-H., Yeh, C.-H. and Shen, C.-C., 2000. A multiobjective model for passenger train services planning: application to Taiwan's high-speed rail line. Transportation Research Part B: Methodological, 34(2), pp. 91-106.

Chen, J.-F., Lin, R.-L. and Liu, Y.-C., 2005. Optimization of an MRT train schedule: reducing maximum traction power by using genetic algorithms. Power Systems, IEEE Transactions on, 20(3), pp. 1366-1372.

Chevrier, R., Pellegrini, P. and Rodriguez, J., 2013. Energy saving in railway timetabling: A bi-objective evolutionary approach for computing alternative running times. Transportation Research Part C: Emerging Technologies, 37, pp. 20-41.

Deb, K., Pratap, A., Agarwal, S. and Meyarivan, T., 2002. A fast and elitist multiobjective genetic algorithm: NSGA-II. Evolutionary Computation, IEEE Transactions on, 6(2), pp. 182197.

Ghoseiri, K., Szidarovszky, F. and Asgharpour, M. J., 2004. A multi-objective train scheduling model and solution. Transportation Research Part B: Methodological, 38(10), pp. 927-952.

Hu, H., Li, K. and Xu, X., 2013. A multi-objective trainscheduling optimization model considering locomotive assignment and segment emission constraints for energy saving. Journal of Modern Transportation, 21(1), pp. 9-16.

Kim, J. H., Bae, Y. K. and Chung, J., 2012. Multi-objective Optimization for Sustainable Road Network Design Problem. In: Int. Conference on Transport, Environment and Civil Engineering, Kuala Lumpur (Malaysia).

Lau, H. C., Agussurja, L., Cheng, S.-F. and Tan, P. J., 2013. A multi-objective memetic algorithm for vehicle resource allocation in sustainable transportation planning. In: Proceedings of the Twenty-Third international joint conference on Artificial Intelligence, AAAI Press.

Nasri, A., Moghadam, M. F. and Mokhtari, H., 2010. Timetable optimization for maximum usage of regenerative energy of braking in electrical railway systems. In: Power Electronics Electrical Drives Automation and Motion (SPEEDAM), 2010 International Symposium on, IEEE.

Saaty, T. L., 1990. How to make a decision: the analytic hierarchy process. European journal of operational research, 48(1), pp. 9-26.

Sels, P., Dewilde, T., Cattrysse, D. and Vansteenwegen, P., 2013. Expected Passenger Travel Time as Objective Function for Train
Schedule Optimization. In: Proceedings of 5th International Seminar on Railway Operations Modelling and Analysis (IAROR): RailCopenhagen2013, May 13-15, Copenhagen, Denmark.

Shaoquan, N., Dingjun, C. and Miaomiao, L., 2009. Research on optimization model of initial schedule of passenger trains based on improved genetic algorithm. In: Intelligent Computation Technology and Automation, 2009. ICICTA'09. Second International Conference on, IEEE.

Su, S., Li, X., Tang, T. and Gao, Z., 2013. A subway train timetable optimization approach based on energy-efficient operation strategy. Intelligent Transportation Systems, IEEE Transactions on, 14(2), pp. 883-893.

Yang, X., Li, X., Gao, Z., Wang, H. and Tang, T., 2013. A cooperative scheduling model for timetable optimization in subway systems. Intelligent Transportation Systems, IEEE Transactions on, 14(1), pp. 438-447.

Yang, X., Ning, B., Li, X. and Tang, T., 2014. A two-objective timetable optimization model in subway systems. Intelligent Transportation Systems, IEEE Transactions on, 15(5), pp. 19131921. 